\title{
Far- and near-field approximation for diffraction radiation
}

\author{
V. Shpakov ${ }^{\text {b,* }}$, S.B. Dabagov ${ }^{\mathrm{a}, \mathrm{b}}$, M. Castellano ${ }^{\mathrm{a}}$, A. Cianchi $^{\mathrm{c}}$, K. Honkavaara $^{\mathrm{d}}$, G. Kube $^{\mathrm{d}}$, E. Chiadroni ${ }^{\text {a }}$ \\ a INFN Laboratori Nazionali di Frascati, Frascati, Italy \\ ${ }^{\mathrm{b}}$ RAS P.N. Lebedev Physical Institute E' NRNU MEPhI, Moscow, Russia \\ "University of Rome "Tor Vergata" E' INFN, Roma, Italy \\ ${ }^{\mathrm{d}}$ Deutsches Elektronen-Synchrotron, Hamburg, Germany
}

\section{A R T I C L E I N F O}

\section{Article history:}

Received 30 November 2012

Received in revised form 13 February 2013

Available online 29 March 2013

\section{Keywords:}

Diffraction radiation

Beam diagnostics

\begin{abstract}
A B S T R A C T
A new beam diagnostic technique based on the Optical Diffraction Radiation Interference (ODRI) has been recently proposed and experimentally investigated. The technique is based on the use of a two slits system. The far field approximation has shown rather accurate correspondence with experimental data when slits are centered with respect to the beam propagation line. Using formulas involving near field we point out in this work that a general treatment is needed with offset larger than $50 \mu \mathrm{m}$.
\end{abstract}

(c) 2013 Elsevier B.V. All rights reserved.

\section{Introduction}

One of the most interesting tasks in high brightness accelerators is the optimization of fourth generation sources of radiation, e.g. Free-Electron Lasers (FEL). At this regard, a precise knowledge of beam parameters, e.g. position, transverse and longitudinal sizes, energy and it's spread, is mandatory. Conventional diagnostics techniques do not always satisfy these requirements. In particular, high brightness, high repetition rate electron beams demand noninvasive, non-intercepting diagnostics for monitoring and measuring beam size in real time. For this reason the techniques based on Diffraction Radiation (DR) [1,2] were proposed and tested [3,4], adopting a single-slit geometry. In order to avoid some problems connected with this geometry, such as the impossibility to separate effects caused by both beam size and beam offset within the slit, a two slits system was proposed [5] and experimentally tested. Since in this system an additional slit is introduced, it becomes necessary to investigate effects caused by new parameters, which appear due to the second slit. In this work we have studied the effects related to the shift of the slits centers relative to each other.

\section{Theory}

Let us consider a system of two slits: the first one is placed perpendicular to the beam line, while the second one is at $45^{\circ}$ (Fig. 1). In order to calculate the distribution of DR on the second slit it is necessary to use near field approximation for FDR from the first slit, which can be written in general form as [4]:

\footnotetext{
* Corresponding author.

E-mail address: shpakov@lnf.infn.it (V. Shpakov).
}

$$
\begin{aligned}
E_{y}^{D R}(x, y)= & \int_{\frac{w_{s l}}{2}+h_{0 y}}^{\infty} d h_{y} \int_{-\infty}^{\infty} E_{y}^{i}\left(h_{x}, h_{y}\right) \frac{\exp (i k s)}{s} d h_{x} \\
& +\int_{-\infty}^{\frac{-w_{s l}}{2}+h_{0 y}} d h_{y} \int_{-\infty}^{\infty} E_{y}^{i}\left(h_{x}, h_{y}\right) \frac{\exp (i k s)}{s} d h_{x},
\end{aligned}
$$

where $s=\sqrt{d^{2}+\left(h_{x}-x\right)^{2}+\left(h_{y}-y\right)^{2}}, h_{x}, h_{y}$ are the coordinates on the slit, $x, y$ are the coordinates on the screen, $d$ is the distance between the slit and screen, $w_{s l}$ is the width of the slit, $h_{0 y}$ takes into account the shift of a slit relative to the beam line, $E_{y}^{i}\left(h_{x}, h_{y}\right)$ is the electromagnetic field of a $i$-particle, and $E_{y}^{\mathrm{DR}}(x, y)$ is the DR amplitude. Both particle field and DR amplitude have the index $y$ since only vertical polarization is considered, according with the experimental setup including a polarizer. Results from numerical calculations are shown in Fig. 2. At the distance of a second slit location, FDR from the first slit represents two separated radiation fluxes from two half-planes of the slit. Indeed, in a two-slit system we look at the interference between backward DR (BDR) from the second slit and reflected forward DR (FDR) from a first one (Fig. 1). It means that depending on the system geometry we may lose some part of radiation from the first slit.

In the experimental setup a lens was used. Therefore, as was shown in [4], the expression for DR from a half plane in such system can be written as:

$$
\begin{aligned}
E_{y}^{D R}\left(h 1, \Theta_{y}\right)= & \frac{i e k}{\pi \gamma} \int_{-\infty}^{\infty} \int_{h 1}^{\infty} d h_{y} d h_{x} \\
& \times \frac{h_{y}}{\sqrt{h_{y}^{2}+h_{x}^{2}}} K_{1}\left(\frac{k}{\gamma} \sqrt{h_{y}^{2}+h_{x}^{2}}\right) e^{-i k\left(h_{y}+h_{0 y}\right) \tan \Theta_{y}}
\end{aligned}
$$

here $\gamma$ is the relativistic factor, $e$ is the electron charge, $\Theta_{y}$ is the direction of radiation spread, $k$ is the wave vector, and $h_{1}$ is the im- 


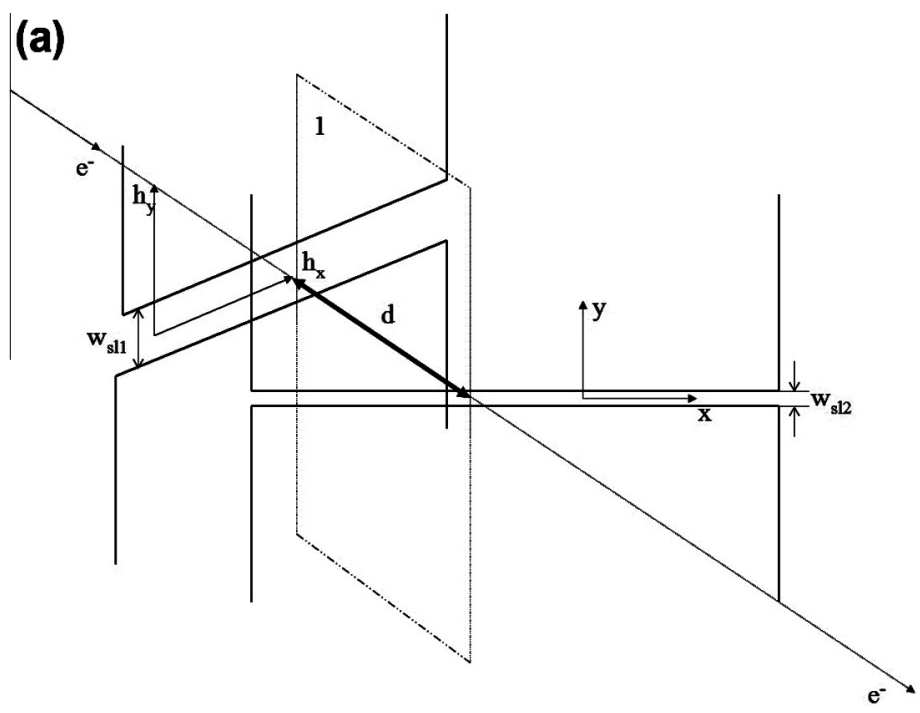

(b)

Fig. 1. Sketch of a two-slits system.
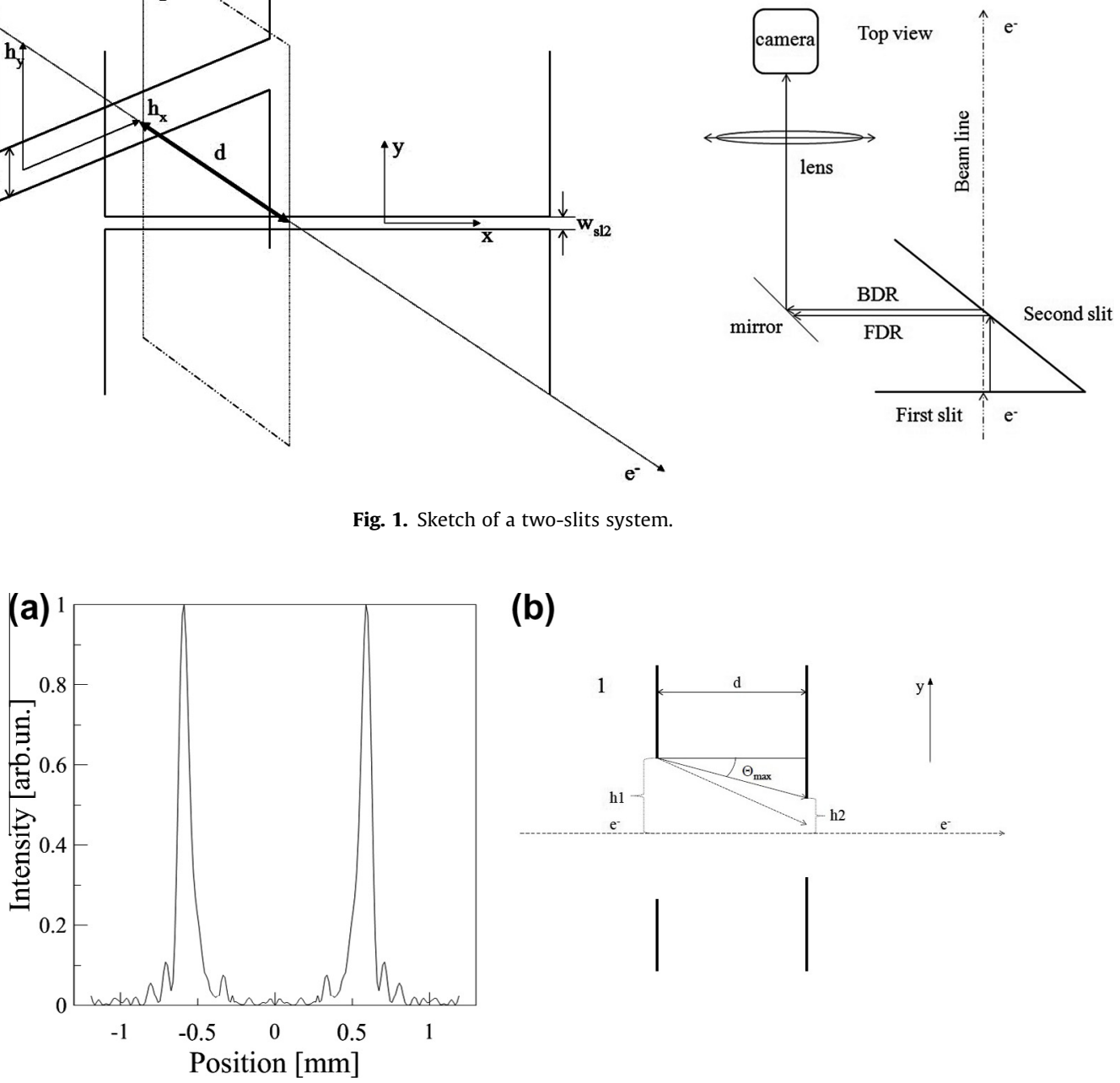

(b)
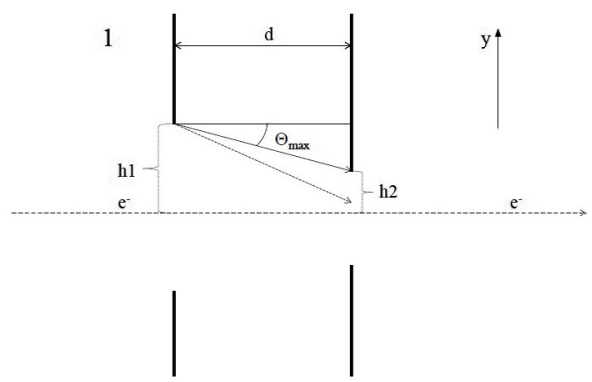

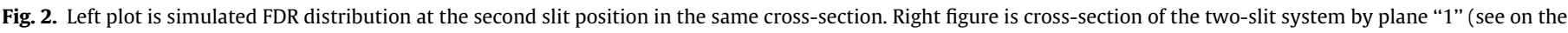
Fig. 1, left).

pact parameter, which depends on the shift of a slit relative to both beam line and particle position inside the bunch. Since we work with FDR reflected from the second slit, part of radiation just passes through into this slit on the second screen. For each geometry we can find the angle of radiation, starting from which FDR is not reflected (Fig. 2).

For the upper half plane the maximum angle, which has to be taken into account, is $\Theta_{\max }=\arctan (h 2-h 1) / d$. Here $h 2$ is the impact parameter for upper half-planes of the second slits, $h 1$ is the coordinate of the point on the first slit, $d$ is the distance between slits. In the same way we can find the maximum angle for lower half-plane. Than we need cut-off all radiation which propagates from point with coordinate $h 1$ in angle bigger than $\Theta_{\max }$. It should be underlined that after some angle the radiation will start to reflect again from the lower (or upper) half-plane of the second slit, but in our work we do not consider so big angles.

In the paper [5] the research for the system with collinear slits was performed and it was shown that additional slit in the system can dramatically change DR angular distribution. In paper [6] changes in DR angular distribution, caused by shifting the slits were investigated. In both cases the far field approximation was used. Also it should be underlined that in experiment the beam divergence was big, and it had to be taken into account. But inte- gration over all possible angles of incidence did not give us adequate agreement with the experiment. In simulations the divergence is always fixed in two times higher than in experiment. We have derived the solution, which is in good agreement with the experimental data. However, this solution is still under discussion, and we hope to dedicate separate paper to this problem.

\section{Calculus}

Let us consider the system where the first slit gap is larger than the second one. We will compare experimental results with numerical calculation without taking into account losses due to reflection [3]. First of all consider the case when the slit are centered or the shift between them is small. Difference between two models is negligible. This fact is easy to understand if to look at Fig. 2. The second slit has the size $0.5 \mathrm{~mm}$, that means we lose radiation falling into the area between 0.25 and $-0.25 \mathrm{~mm}$. The part of radiation, which falls in this region, is negligible. If we shift the first slit for $300 \mu \mathrm{m}$, one of the half-planes of the first slit has impact the parameter even less than that for the second slit. In this case we cannot neglect the losses inside the second slit. In Fig. 3 you can see the difference between two approximations. Fig. 4 . 


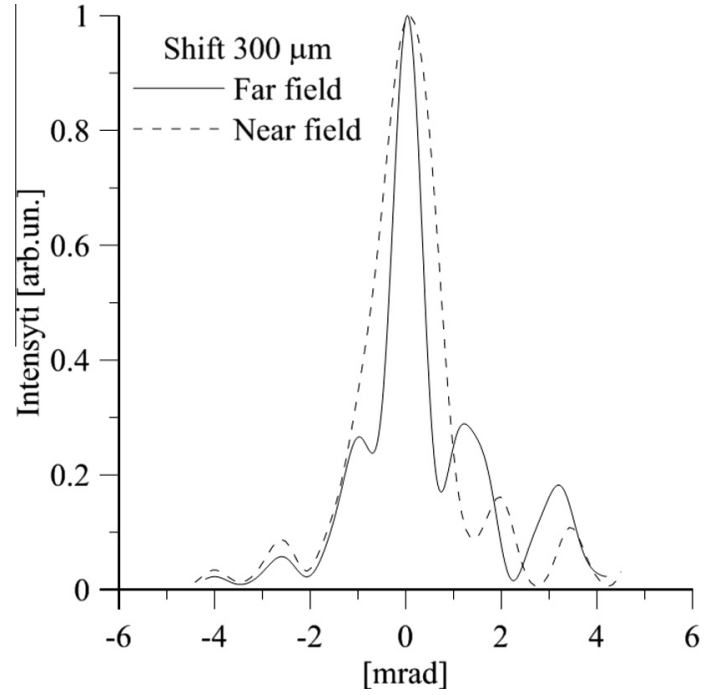

Fig. 3. Simulated angular distributions of DR. Solid line accords to far-field calculations, dashed line - near field. The shift between the slits centers is $300 \mu \mathrm{m}$.

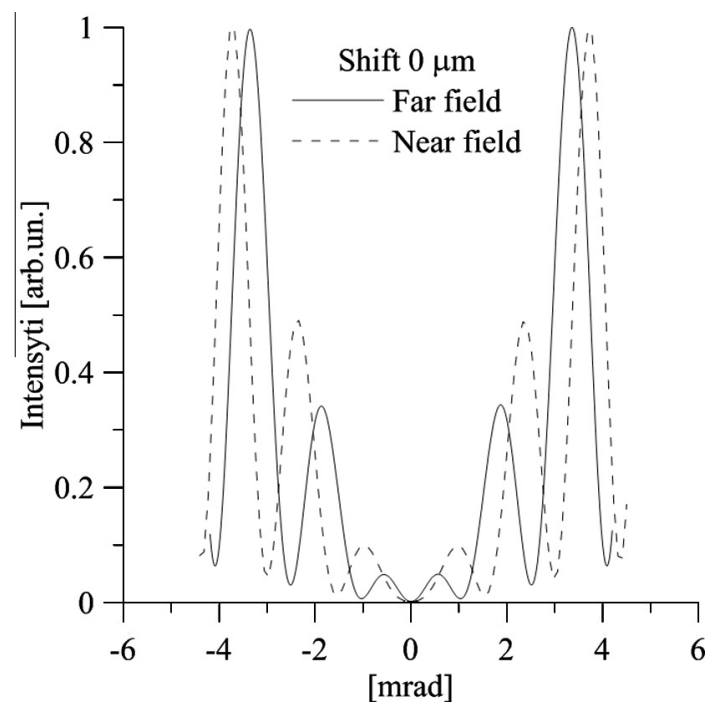

Fig. 4. Simulated angular distributions of DR for the system with equivalent slits. The shift between the slits centers is $0 \mu \mathrm{m}$.

\section{Experimental results}

Experiments were performed at the FLASH free-electron laser test facility at DESY (Hamburg). The beam energy was $1 \mathrm{GeV}$, while the size of the first slit was $1 \mathrm{~mm}$. Second slit center was placed at $2.5 \mathrm{~cm}$ and its size was $0.5 \mathrm{~mm}$.

Experiments were performed for two cases. First one for the wavelength of DR $800 \mathrm{~nm}$. Center of the second slit were fixed in $65 \mu \mathrm{m}$ from the electron beam trajectory. The first slit was movable in "y" direction (see on the Fig. 2 right). Positive values of the shift correspond to the displacement in the direction of positive angles, and contrary for negative values. Initial position of the first slit center was $35 \mu \mathrm{m}$ (Fig. 5) and final position $-85 \mu \mathrm{m}$. For both measurements beam size was $87 \mu \mathrm{m}$ and divergence $-64 \mu \mathrm{rad}$. On the plots solid curve accords to model where losses because of reflection are not taken into account, and on the contrary for the dashed curve. The experimental data are given by empty circles.

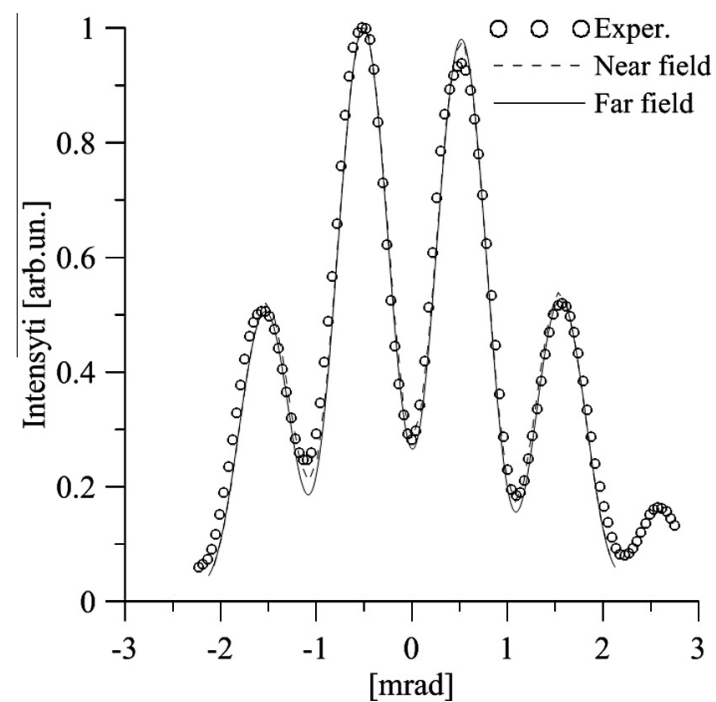

Fig. 5. Angular distributions of $D R$ for the case of $800 \mathrm{~nm}$ wavelength. The shift between the slits centers $35 \mu \mathrm{m}$.

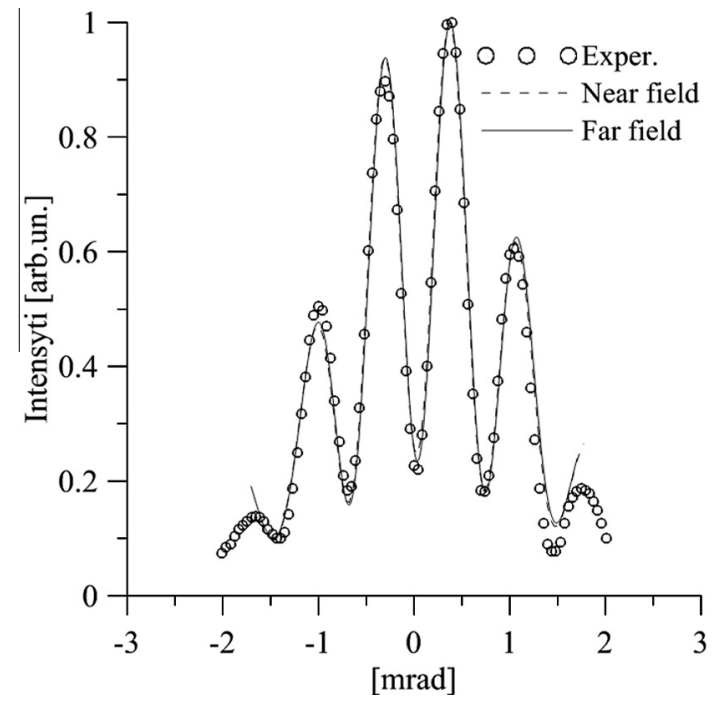

Fig. 6. Angular distributions of the DR for the case of $800 \mathrm{~nm}$ wavelength.The shift between the slits centers $100 \mu \mathrm{m}$.

In second experiments the wavelength of DR was $500 \mathrm{~nm}$. The center of second slit was in $10 \mu \mathrm{m}$ from the electron beam trajectory. Initial position of the first slit center was $50 \mu \mathrm{m}$, while final position $-100 \mu \mathrm{m}$ (Fig. 6). In this case for both measurements the beam size was $46 \mu \mathrm{m}$ and the divergence - $66 \mu \mathrm{rad}$.

\section{Conclusion}

We have shown that for the geometry of a two-slit system, used in experiments, the radiation losses can be negligible. For such case we can apply far-field approximation with good accuracy. However, we also have shown that for the big shifts (comparable with the size of a slit) between the slits centers, the radiation losses become important. For the system where slits has the same size such effects could not be excluded at all, since significant part of radiation is lost at the beginning. 


\section{Acknowledgement}

V.Sh would like to express special gratitude to the Scholarship of the President of Russian Federation for support in conducting research.

\section{References}

[1] M.L. Ter-Mikaelian, High-energy Electromagnetic Processes in Condensed Media, Wiley, Interscience, New York, 1972.
[2] A.P. Potylitsyn, M.I. Ryazanov, M.N. Strikhanov, A.A. Tishchenko, Diffraction Radiation from Relativistic Particles. STMP 239, Springer, Berlin Heidelberg, 2010.

[3] E. Chiadroni, M. Castellano, A. Cianchi, K. Honkavaara, G. Kube, V. Merlo, F. Stella, Nucl. Instrum. Meth. Phys. Res. B 266 (2008) 3789.

[4] P. Karataev, S. Akari, A. Aryshev, G. Naumenko, A.P. Potylitsyn, N. Terunuma, J. Urakawa, Phys. Rev. 11 (2008) 032804.

[5] A. Cianchi, M. Castellano, L. Catani, E. Chiadroni, K. Honkavaara, G. Kube, Phys. Rev. 14 (2011) 102803.

[6] V. Shpakov, S.B. Dabagov, Russ. Phy. J. 11 (2012) 88-93. 